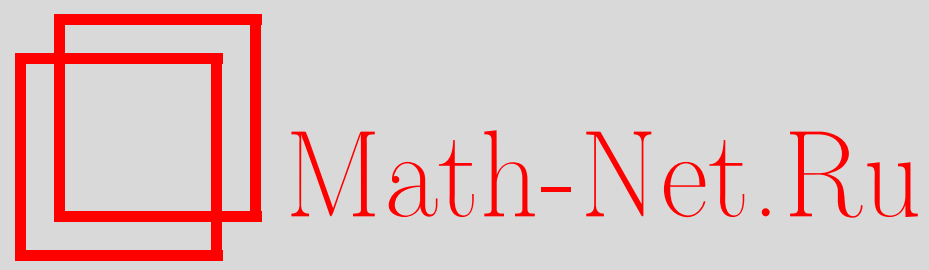

А. Г. Савушкина, О группе сопрягающих автоморфизмов свободной группы, Матем. заметки, 1996, том 60, выпуск 1, 92-108

DOI: https://doi.org/10.4213/mzm1806

Использование Общероссийского математического портала MathNet.Ru подразумевает, что вы прочитали и согласны с пользовательским соглашением

http://www . mathnet.ru/rus/agreement

Параметры загрузки:

IP: 18.209 .158 .208

26 апреля 2023 г., 06:11:57

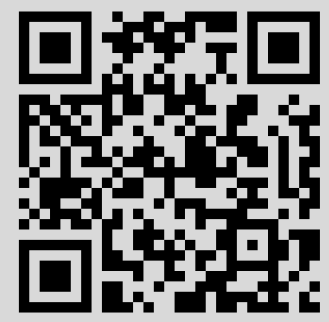


том 60 выпуск 1 июль 1996

\section{О ГРУППЕ СОПРЯГАЮЩИХ АВТОМОРФИЗМОВ СВОБОДНОЙ ГРУППЫ}

\section{А. Г. Савушкина}

Пусть $F_{n},(n \geqslant 2)$ - свободная группа со свободными образующими $a_{1}, \ldots, a_{n}$ и пусть Aut $F_{n}$ - группа автоморфизмов свободной группы $F_{n}$. Автоморфизм из групшы Aut $F_{n}$ назьвается сопрягающим автоморфизмом, если он отображает каждую образующую $a_{p}$ в слово вида $W_{p}^{-1}\left(a_{1}, \ldots, a_{n}\right) a_{i_{p}} W_{p}\left(a_{1}, \ldots, a_{n}\right)$. Группа сопрягающих автоморфизмов обозначается через $\mathbf{C}_{n}$. При задании автоморфизмов неподвижные образуюшие не выписываются. Автоморфизм из группы $\mathbf{C}_{n}$ называется сопрягающим базис автоморфизмом (см. [1]), если он отображает каждую образующую $a_{p}$ в слово вида $W_{p}^{-1}\left(a_{1}, \ldots, a_{n}\right) a_{p} W_{p}\left(a_{1}, \ldots, a_{n}\right)$. Группа сопрягающих базис автоморфизмов $\mathfrak{M}_{n}$ порождается автоморфизмами (см. [2])

$$
\varepsilon_{i, j}: a_{i} \rightarrow a_{j}^{-1} a_{i} a_{j} \quad(1 \leqslant i, j \leqslant n, i \neq j)
$$

и определяюшими соотношениями (см. [1])

$$
\begin{aligned}
\varepsilon_{i, j} \varepsilon_{k, l} & =\varepsilon_{k, l} \varepsilon_{i, j} \quad(i \neq k, i \neq l, j \neq k), \\
\varepsilon_{i, k} \varepsilon_{i, j} \varepsilon_{k, i} & =\varepsilon_{k, j} \varepsilon_{i, j} \varepsilon_{i, k} .
\end{aligned}
$$

Симметрическая группа подстановок $\mathfrak{S}_{n}$ является подгруппой групы $\mathbf{C}_{n}$. Она порождается автоморфизмами

$$
\alpha_{i}:\left\{\begin{array}{l}
a_{i} \rightarrow a_{i+1} \\
a_{i+1} \rightarrow a_{i}
\end{array} \quad(i=1, \ldots, n-1),\right.
$$

и определяющими соотношениями (см., например, [3])

$$
\begin{gathered}
\alpha_{i}^{2}=1, \quad \alpha_{i} \alpha_{i+1} \alpha_{i}=\alpha_{i+1} \alpha_{i} \alpha_{i+1} \quad(1 \leqslant i \leqslant n-2), \\
\alpha_{i} \alpha_{k}=\alpha_{k} \alpha_{i} \quad(1 \leqslant i, k \leqslant n-1, \quad|i-k|>1) .
\end{gathered}
$$

Подгруппа групы $\mathbf{C}_{n}$, состоящая из тех автоморфизмов, которые отображают на себя произведение $a_{1} a_{2} \cdots a_{n}$, является группой кос $\mathfrak{B}_{n}$ (см., например, [4]). Она порождается автоморфизмами

$$
\sigma_{i}:\left\{\begin{array}{l}
a_{i} \rightarrow a_{i+1} \\
a_{i+1} \rightarrow a_{i+1}^{-1} a_{i} a_{i+1}
\end{array} \quad(i=1, \ldots, n-1),\right.
$$


и определяющими соотношениями (см. [5])

$$
\begin{gathered}
\sigma_{i} \sigma_{i+1} \sigma_{i}=\sigma_{i+1} \sigma_{i} \sigma_{i+1} \quad(i=1, \ldots, n-2) \\
\sigma_{i} \sigma_{j}=\sigma_{j} \sigma_{i} \quad(i, j=1, \ldots, n-1, \quad|i-j|>1)
\end{gathered}
$$

Подгруппа групшы кос $\mathfrak{B}_{n}$, порожденная автоморфизмами

$$
\rho_{i, j}=\sigma_{j-1} \sigma_{j-2} \cdots \sigma_{i+1} \sigma_{i}^{2} \sigma_{i+1} \cdots \sigma_{j-2} \sigma_{j-1} \quad(1 \leqslant i<j \leqslant n)
$$

является группой крашеньг кос $\mathfrak{N}_{n}$ (см. [6]).

В настоящей работе доказано, что группа сопрягающих автоморфизмов $\mathbf{C}_{n}$ является полупрямьм произведением (нормальной) подгрупшы $\mathfrak{M}_{n}$ на подгруппу $\mathfrak{S}_{n}$. Приведено задание группы $\mathbf{C}_{n}$ через образуюшие групп $\mathfrak{B}_{n}$ и $\mathfrak{S}_{n}$. Указаны выражения образуюших групшы $\mathfrak{N}_{n}$ через образующие групшы $\mathfrak{M}_{n}$. Групшы $\mathbf{C}_{n}$ и $\mathfrak{M}_{n}$ имеют тривиальные центры. Указаны необходимые и достаточные условия принадлежности автоморфизма коммутанту $\mathbf{C}_{n}^{\prime}$ групшы $\mathbf{C}_{n}$ и коммутанту $\mathfrak{M}_{n}^{\prime}$ группы $\mathfrak{M}_{n}$. Доказано, что $\mathfrak{M}_{n}^{\prime} \cap \mathfrak{B}_{n}^{\prime}=\mathfrak{N}_{n}^{\prime}$. Показано, что ядро представления Бурау групшы кос $\mathfrak{B}_{n}$ матрицами $n$-го порядка (с элементами из кольца многочленов Лорана от одной переменной с цельми коэффициентами) принадлежит $\mathfrak{N}_{n}^{\prime}$, и всякий элемент из ядра представления Гасснер грушы крашеных кос $\mathfrak{N}_{n}$ матрицами $n$-го порядка (с элементами из кольца многочленов Лорана от $n$ переменных с целыми коэффициентами) при выдергивании любой нити переходит в некоторьй элемент ядра представления Гасснер группы $\mathfrak{N}_{n-1}$. Эти результаты усиливают оба утверждения теоремы 3.14 Бирман [7].

Лемма 1. Группа $\mathbf{C}_{n}$ порождается автоморфизмами (1), (3) и определяющими соотношениями (2), (4) и

$$
\begin{gathered}
\varepsilon_{i, j} \alpha_{k}=\alpha_{k} \varepsilon_{i, j}, \quad k \neq i, i-1, j, j-1, \\
\varepsilon_{i, j} \alpha_{i}=\alpha_{i} \varepsilon_{i+1, j}, \quad \varepsilon_{i, j} \alpha_{j}=\alpha_{j} \varepsilon_{i, j+1}, \quad \varepsilon_{i, i+1} \alpha_{i}=\alpha_{i} \varepsilon_{i+1, i}
\end{gathered}
$$

ДокАЗАТЕЛЬСТво. Пусть $C=a_{i} \rightarrow W_{i}^{-1} a_{\gamma_{i}} W_{i}(i=1, \ldots, n)$, где $\left(\begin{array}{c}1 \ldots n \\ \gamma_{1} \ldots \gamma_{n}\end{array}\right)$ - подстановка, - произвольньй автоморфизм из групшы $\mathbf{C}_{n}$. Автоморфизм $Q=a_{\gamma_{j}} \rightarrow a_{j}(j=1, \ldots, n)$ может быть представлен, как слово в алфавите $\alpha_{1}, \ldots, \alpha_{n-1}$. Если $Q=R\left(\alpha_{1}, \ldots, \alpha_{n-1}\right)$, то автоморфизм $C R$ является сопрягающим базис и по теореме Хэмфри (см. [2]) порождается образуюшими $\left\{\varepsilon_{i, j}\right\}$. Если $C R=T\left(\varepsilon_{i, j}\right)$, то $C=T\left(\varepsilon_{i, j}\right) R^{-1}\left(\alpha_{1}\right.$, $\left.\ldots, \alpha_{n-1}\right)$, т.е. автоморфизм $C$ порождается автоморфизмами $\left\{\varepsilon_{i, j}\right\},\left\{\alpha_{k}\right\}$.

То, что $(2),(4),(8)$ вьполняются в групе $\mathbf{C}_{n}$ проверяется из определений автоморфизмов $\left\{\varepsilon_{i, j}\right\}$ и $\left\{\alpha_{k}\right\}$. Для любой пары образующих $\alpha_{k}$ и $\varepsilon_{i, j}$ в системе (8) найдется соотношение вида $\alpha_{k} \varepsilon_{i, j}=\varepsilon_{i_{1}, j_{1}} \alpha_{k}$ для некоторого $\varepsilon_{i_{1}, j_{1}}$. "Передвигая" в тождественном автоморфизме $P\left(\varepsilon_{i, j}, \alpha_{i}\right)$ все $\alpha_{i}$ в конец слова, переведем соотношениями $(8)$ слово $P\left(\varepsilon_{i, j}, \alpha_{i}\right)$ в слово 
$R\left(\varepsilon_{i, j}\right) V\left(\alpha_{i}\right)$. Поскольку $V\left(\alpha_{i}\right)=R^{-1}\left(\varepsilon_{i, j}\right)$, то автоморфизм $V\left(\alpha_{i}\right)$ является сопрягаюшим базис и, следовательно, переводится в единицу соотношениями (4). Автоморфизм $R\left(\varepsilon_{i, j}\right)$ также тождественный и по теореме Маккула (см. [1]) переводится в единицу соотношениями (2).

Рассмотрим список автоморфизмов

$$
\beta_{i}: a_{i} \rightarrow a_{i+1}^{-1} a_{i} a_{i+1} \quad(i=1, \ldots, n-1) .
$$

Автоморфизмы $\left\{\alpha_{k}\right\}$ и $\left\{\beta_{k}\right\}$ назовем элементарными.

Из определения автоморфизмов следует равенство

$$
\beta_{i}=\varepsilon_{i, i+1} \quad(i=1, \ldots, n-1) .
$$

Равенства

$$
\begin{aligned}
& \varepsilon_{i, j}=\alpha_{j-1} \alpha_{j-2} \cdots \alpha_{i+1} \beta_{i} \alpha_{i+1} \cdots \alpha_{j-2} \alpha_{j-1}, \quad(i<j), \\
& \varepsilon_{j, i}=\alpha_{j-1} \alpha_{j-2} \cdots \alpha_{i+1} \alpha_{i} \beta_{i} \alpha_{i} \alpha_{i+1} \cdots \alpha_{j-2} \alpha_{j-1} \quad(i<j)
\end{aligned}
$$

показывают, каким образом автоморфизмы $\varepsilon_{i, j}$ выражаются через элементарные автоморфизмы. Равенства (11) проверяются из определений автоморфизмов.

Лемма 2. Группа $\mathbf{C}_{n}$ порожсдается автоморфизмами (3), (9) и определяющими соотношениями (4) и

$$
\begin{gathered}
\beta_{i} \beta_{j}=\beta_{j} \beta_{i}, \quad|i-j|>1, \\
\alpha_{i} \beta_{j}=\beta_{j} \alpha_{i}, \quad|i-j|>1, \quad \alpha_{i} \beta_{i+1} \alpha_{i}=\alpha_{i+1} \beta_{i} \alpha_{i+1}, \\
\left(\alpha_{i} \beta_{i+1}\right)^{2}=\left(\beta_{i+1} \alpha_{i}\right)^{2}, \quad\left(\alpha_{i} \beta_{i+1}\right)^{2} \beta_{i}=\beta_{i}\left(\alpha_{i} \beta_{i+1}\right)^{2} .
\end{gathered}
$$

ДокАЗАТЕЛЬСтво. По лемме 1 группа $\mathbf{C}_{n}$ порождается автоморфизмами $\left\{\varepsilon_{i, j}\right\},\left\{\alpha_{k}\right\}$ и определяюшими соотношениями $(2),(4),(8)$. Добавив образуюшие $\left\{\beta_{k}\right\}$ и определяюшие соотношения (10) получим изоморфную группу. Из определений автоморфизмов $\alpha_{i}, \beta_{i}, \varepsilon_{i, j}$ следует, что равенства $(11),(12),(13)$ вьполняются в группе $\mathbf{C}_{n}$. Следовательно, по лемме 1 они вьводимы из соотношений $(2),(4),(8),(10)$, и группа, задаваемая образуюшими $\left\{\varepsilon_{i, j}\right\},\left\{\alpha_{k}\right\},\left\{\beta_{k}\right\}$ и определяюшими соотношениями $(2),(4),(8),(10),(11),(12),(13)$, изоморфна групе $\mathbf{C}_{n}$. В дальнейшем мы покажем, что соотношения (2), (8) выводятся из (4), (10), (11), (12), (13). Соотношения

$$
\begin{array}{ll}
\alpha_{j-1} \alpha_{j-2} \cdots \alpha_{i+1} \beta_{i} \alpha_{i+1} \cdots \alpha_{j-2} \alpha_{j-1} & \\
\quad=\alpha_{i} \alpha_{i+1} \cdots \alpha_{j-2} \beta_{j-1} \alpha_{j-2} \cdots \alpha_{i+1} \alpha_{i} & (i<j), \\
\alpha_{j-1} \alpha_{j-2} \cdots \alpha_{i} \beta_{i} \alpha_{i} \cdots \alpha_{j-2} \alpha_{j-1} & \\
\quad=\alpha_{i} \alpha_{i+1} \cdots \alpha_{j-1} \beta_{j-1} \alpha_{j-1} \cdots \alpha_{i+1} \alpha_{i} & (i<j)
\end{array}
$$

выводятся из соотношений $(4),(13)$, поэтому ими можно пользоваться. 
Воспользовавшись соотношениями (11), запишем соотношения (8) в алфавите $\left\{\alpha_{k}\right\},\left\{\beta_{k}\right\}$. "Передвигая" при помоши соотношений (4) образующие $\alpha_{k}$ внутрь выражений

$$
\begin{gathered}
\alpha_{j-1} \alpha_{j-2} \cdots \alpha_{i+1} \beta_{i} \alpha_{i+1} \cdots \alpha_{j-2} \alpha_{j-1}, \\
\alpha_{j-1} \alpha_{j-2} \cdots \alpha_{i+1} \alpha_{i} \beta_{i} \alpha_{i} \alpha_{i+1} \cdots \alpha_{j-2} \alpha_{j-1}
\end{gathered}
$$

и используя соотношения (13), мы приведем полученные равенства к тождествам.

Для доказательства первого соотношения системы (2) сначала необходимо доказать выводимость равенства

$$
\varepsilon_{p, q} \beta_{i}=\beta_{i} \varepsilon_{p, q} \quad(i \neq p, q, p-1) .
$$

Если $q<i<p$, то, воспользовавшись соотношениями (11), (15), получим равенство

$$
\begin{aligned}
\alpha_{q} \alpha_{q+1} \cdots \alpha_{p-1} \beta_{p-1} \alpha_{p-1} & \cdots \alpha_{q+1} \alpha_{q} \beta_{i} \\
= & \beta_{i} \alpha_{q} \alpha_{q+1} \cdots \alpha_{p-1} \beta_{p-1} \alpha_{p-1} \cdots \alpha_{q+1} \alpha_{q}
\end{aligned}
$$

выводимое из соотношений (12), (13).

Если $q<p<i$, то, воспользовавшись соотношениями (11), (15), получим равенство

$$
\begin{aligned}
\alpha_{q} \alpha_{q+1} \cdots \alpha_{p-1} \beta_{p-1} \alpha_{p-1} & \cdots \alpha_{q+1} \alpha_{q} \beta_{i} \\
= & \beta_{i} \alpha_{q} \alpha_{q+1} \cdots \alpha_{p-1} \beta_{p-1} \alpha_{p-1} \cdots \alpha_{q+1} \alpha_{q}
\end{aligned}
$$

выводимое из соотношений (12), (13).

Если $i<q<p$ и $i \neq q-1$, то, воспользовавшись соотношениями (11), (15), получим равенство

$$
\begin{aligned}
\alpha_{q} \alpha_{q+1} \cdots \alpha_{p-1} \beta_{p-1} \alpha_{p-1} & \cdots \alpha_{q+1} \alpha_{q} \beta_{i} \\
= & \beta_{i} \alpha_{q} \alpha_{q+1} \cdots \alpha_{p-1} \beta_{p-1} \alpha_{p-1} \cdots \alpha_{q+1} \alpha_{q}
\end{aligned}
$$

выводимое из соотношений (12), (13).

Если $i<q<p$ и $i=q-1$, то, воспользовавшись последовательно первым, вторым и третьим равенством соотношений (13), получим равенство

$$
\begin{aligned}
& \alpha_{p-1} \alpha_{p-2} \cdots \alpha_{q} \alpha_{q-1} \beta_{q} \alpha_{q-1} \beta_{q} \alpha_{q} \alpha_{q+1} \cdots \alpha_{p-2} \alpha_{p-1} \\
& =\beta_{q-1} \alpha_{p-1} \alpha_{p-2} \cdots \alpha_{q} \beta_{q} \alpha_{q} \cdots \alpha_{p-2} \alpha_{p-1},
\end{aligned}
$$

выводимое из соотношений (13).

Если $p<i<q$, то, воспользовавшись соотношениями (11), получим равенство

$$
\begin{aligned}
& \alpha_{q-1} \alpha_{q-2} \cdots \alpha_{p+1} \beta_{p} \alpha_{p+1} \cdots \alpha_{q-2} \alpha_{q-1} \beta_{i} \\
& \quad=\beta_{i} \alpha_{q-1} \alpha_{q-2} \cdots \alpha_{p+1} \beta_{p} \alpha_{p+1} \cdots \alpha_{q-2} \alpha_{q-1}
\end{aligned}
$$


выводимое из соотношений (12), (13).

Если $p<q<i$, то, воспользовавшись соотношениями (11), получим равенство

$$
\begin{aligned}
& \alpha_{q-1} \alpha_{q-2} \cdots \alpha_{p+1} \beta_{p} \alpha_{p+1} \cdots \alpha_{q-2} \alpha_{q-1} \beta_{i} \\
& \quad=\beta_{i} \alpha_{q-1} \alpha_{q-2} \cdots \alpha_{p+1} \beta_{p} \alpha_{p+1} \cdots \alpha_{q-2} \alpha_{q-1},
\end{aligned}
$$

выводимое из соотношений (12), (13).

Если $i<p<q$, то, воспользовавшись соотношениями (11), получим равенство

$$
\begin{aligned}
& \alpha_{q-1} \alpha_{q-2} \cdots \alpha_{p+1} \beta_{p} \alpha_{p+1} \cdots \alpha_{q-2} \alpha_{q-1} \beta_{i} \\
& \quad=\beta_{i} \alpha_{q-1} \alpha_{q-2} \cdots \alpha_{p+1} \beta_{p} \alpha_{p+1} \cdots \alpha_{q-2} \alpha_{q-1},
\end{aligned}
$$

выводимое из соотношений (12), (13).

Докажем первое равенство соотношений (2). Используя соотношения (11), перепишем его в виде

$$
\begin{aligned}
& \alpha_{j-1} \alpha_{j-2} \cdots \alpha_{i+1} \beta_{i} \alpha_{i+1} \cdots \alpha_{j-2} \alpha_{j-1} \varepsilon_{k, l} \\
& \quad=\varepsilon_{k, l} \alpha_{j-1} \alpha_{j-2} \cdots \alpha_{i+1} \beta_{i} \alpha_{i+1} \cdots \alpha_{j-2} \alpha_{j-1} \quad(i<j), \\
& \quad \alpha_{i-1} \alpha_{i-2} \cdots \alpha_{j} \beta_{j} \alpha_{j} \cdots \alpha_{i-2} \alpha_{i-1} \varepsilon_{k, l} \\
& \quad=\varepsilon_{k, l} \alpha_{i-1} \alpha_{i-2} \cdots \alpha_{j} \beta_{j} \alpha_{j} \cdots \alpha_{i-2} \alpha_{i-1} \quad(j>i) .
\end{aligned}
$$

Пользуясь соотношениями (8), которые, как мы уже доказали, вьводимы из (4), (10), (11), (12), (13), “передвинем" образующие $\left\{\alpha_{k}\right\}$ соответственно в начала и конщы соотношений и сократим их. В результате получим соотношение (16).

Для доказательства второго соотношения системы (2) докажем вьводимость равенств

$$
\begin{aligned}
\varepsilon_{i, j+1} \beta_{j} \varepsilon_{i, j} & =\varepsilon_{i, j} \beta_{j} \varepsilon_{i, j+1} & & (i<j), \\
\varepsilon_{i, j} \varepsilon_{i+1, j} \beta_{i} & =\beta_{i} \varepsilon_{i+1, j} \varepsilon_{i, j} & & (j<i), \\
\beta_{i} \varepsilon_{j, i+1} \varepsilon_{i, j} & =\varepsilon_{i, j} \varepsilon_{j, i+1} \beta_{i} & & (j<i) .
\end{aligned}
$$

Используя соотношения (11), перепишем соотношение (17) в алфавите $\left\{\alpha_{k}\right\},\left\{\beta_{k}\right\}$. Применим соотношение (14) и сократим слева и справа образуюшие $\left\{\alpha_{k}\right\}$. Затем, пользуясь соотношениями (13) и (4), приведем его к виду $\alpha_{j-1} \beta_{j} \alpha_{j-1} \beta_{j} \beta_{j-1}=\beta_{j-1} \beta_{j} \alpha_{j-1} \beta_{j} \alpha_{j-1}$, т.е. к четвертому равенству соотношения (13).

Используя соотношения (11), перепишем соотношения (18) в алфавите $\left\{\alpha_{k}\right\},\left\{\beta_{k}\right\}$. Из соотношений (4) имеем

$$
\begin{aligned}
\alpha_{j} \alpha_{j+1} \cdots \alpha_{i-2} \alpha_{i-1} \alpha_{i} \alpha_{i-1} \alpha_{i-2} & \cdots \alpha_{j+1} \alpha_{j} \\
= & \alpha_{i} \alpha_{i-1} \cdots \alpha_{j+1} \alpha_{j} \alpha_{j+1} \cdots \alpha_{i-1} \alpha_{i} .
\end{aligned}
$$


Используем второе равенство соотношения (13) и соотношения (4). Применив соотношение (15), сократим слева и справа образующие $\left\{\alpha_{k}\right\}$. Затем, пользуясь соотношениями (13) и (4), приведем его к виду $\alpha_{j} \beta_{j+1} \alpha_{j} \beta_{j+1} \beta_{j}=\beta_{j} \beta_{j+1} \alpha_{j} \beta_{j+1} \alpha_{j}$, т.е. к четвертому равенству соотношения (13).

Используя соотношения (11), перепишем соотношение (19) в алфавите $\left\{\alpha_{k}\right\},\left\{\beta_{k}\right\}$. Применив соотношения (14) и (15), сократим в алфавите $\left\{\alpha_{k}\right\},\left\{\beta_{k}\right\}$. Применив соотношения (14) и (15), сократим образуюшие $\left\{\alpha_{k}\right\}$. Затем, пользуясь соотношениями (13) и (4), приведем его к виду $\alpha_{i-1} \beta_{i} \alpha_{i-1} \beta_{i} \beta_{i-1}=\beta_{i-1} \beta_{i} \alpha_{i-1} \beta_{i} \alpha_{i-1}$, т.е. к четвертому равенству соотношения (13).

Доказательство второго соотношения системы (2) разбивается на шесть случаев в зависимости от величин $i, j, k$. Если $i<j<k$, то, воспользовавшись соотношением (11), получим

$$
\begin{aligned}
\varepsilon_{i, j} \alpha_{k-1} \alpha_{k-2} \cdots \alpha_{j+1} \alpha_{j} \beta_{j} \alpha_{j+1} \alpha_{j+1} \cdots & \\
& \alpha_{k-2} \alpha_{k-1} \alpha_{k-1} \alpha_{k-2} \cdots \alpha_{i+1} \beta_{i} \alpha_{i+1} \cdots \alpha_{k-2} \alpha_{k-1} \\
= & \alpha_{k-1} \alpha_{k-2} \cdots \alpha_{i+1} \beta_{i} \alpha_{i+1} \cdots \\
& \alpha_{k-2} \alpha_{k-1} \alpha_{k-1} \alpha_{k-2} \cdots \alpha_{j+1} \alpha_{j} \beta_{j} \alpha_{j} \alpha_{j+1} \cdots \alpha_{k-2} \alpha_{k-1} \varepsilon_{i, j} .
\end{aligned}
$$

Применив соотношения (4), сократим образующие $\left\{\alpha_{k}\right\}$. Пользуясь соотношениями (8), “передвинем" образующие соответственно в начало и в конец соотношений и сократим их. Домножим равенство слева и справа на $\alpha_{j}$ и сократим $\alpha_{j}^{2}$. По третьему равенству соотношения (8) и соотношению (11) получим соотношение (17).

Если $i<k<j$, то, воспользовавшись соотношением (11), получим

$$
\begin{aligned}
\alpha_{j-1} \alpha_{j-2} \cdots \alpha_{i+1} \beta_{i} \alpha_{i+1} \cdots \alpha_{j-2} \alpha_{j-1} \alpha_{j-1} \alpha_{j-2} \cdots \\
\quad \alpha_{k+1} \beta_{k} \alpha_{k+1} \cdots \alpha_{j-2} \alpha_{j-1} \varepsilon_{i, k} \\
=\varepsilon_{i, k} \alpha_{j-1} \alpha_{j-2} \cdots \alpha_{k+1} \beta_{k} \alpha_{k+1} \cdots \\
\quad \alpha_{j-2} \alpha_{j-1} \alpha_{j-1} \alpha_{j-2} \cdots \alpha_{i+1} \beta_{i} \alpha_{i+1} \cdots \alpha_{j-2} \alpha_{j-1} .
\end{aligned}
$$

Применив соотношения (4), сократим образующие $\left\{\alpha_{k}\right\}$. Пользуясь соотношениями (8), "передвинем" образующие $\left\{\alpha_{k}\right\}$ соответственно в начало и в конец соотношений и сократим их. По соотношению (11) получим равенство (17).

Если $j<i<k$, то, воспользовавшись соотношением (11), получим

$$
\begin{aligned}
& \varepsilon_{i, j} \alpha_{k-1} \alpha_{k-2} \cdots \alpha_{j+1} \alpha_{j} \beta_{j} \alpha_{j} \alpha_{j+1} \cdots \\
& \quad \alpha_{k-2} \alpha_{k-1} \alpha_{k-1} \alpha_{k-2} \cdots \alpha_{i+1} \beta_{i} \alpha_{i+1} \cdots \alpha_{k-2} \alpha_{k-1} \\
& =\alpha_{k-1} \alpha_{k-2} \cdots \alpha_{i+1} \beta_{i} \alpha_{i+1} \cdots \\
& \quad \alpha_{k-2} \alpha_{k-1} \alpha_{k-1} \alpha_{k-2} \cdots \alpha_{j+1} \alpha_{j} \beta_{j} \alpha_{j} \alpha_{j+1} \cdots \alpha_{k-2} \alpha_{k-1} \varepsilon_{i, j} .
\end{aligned}
$$


Применив соотношения (4), сократим образующие $\left\{\alpha_{k}\right\}$. Пользуясь соотношениями (8), “передвинем" образующие $\left\{\alpha_{k}\right\}$ соответственно в начало и в конец соотношений и сократим их. По соотношению (11) получим равенство (18).

Если $j<k<i$, то, воспользовавшись соотношением (11), получим

$$
\begin{aligned}
\alpha_{i-1} \alpha_{i-2} \cdots \alpha_{j+1} \alpha_{j} \beta_{j} \alpha_{j} \alpha_{j+1} \cdots & \\
& \alpha_{i-2} \alpha_{i-1} \varepsilon_{k, j} \alpha_{i-1} \alpha_{i-2} \cdots \alpha_{k+1} \alpha_{k} \beta_{k} \alpha_{k} \alpha_{k+1} \cdots \alpha_{i-2} \alpha_{i-1} \\
= & \alpha_{i-1} \alpha_{i-2} \cdots \alpha_{k+1} \alpha_{k} \beta_{k} \alpha_{k} \alpha_{k+1} \cdots \\
& \alpha_{i-2} \alpha_{i-1} \varepsilon_{k, j} \alpha_{i-1} \alpha_{i-2} \cdots \alpha_{j+1} \alpha_{j} \beta_{j} \alpha_{j} \alpha_{j+1} \cdots \alpha_{i-2} \alpha_{i-1} .
\end{aligned}
$$

Сократим слева и справа образуюшие $\left\{\alpha_{k}\right\}$. Пользуясь соотношениями (8), "передвинем" образующие $\left\{\alpha_{k}\right\}$ и сократим их. По второму равенству соотношения (8) и соотношению (11) получим соотношение (18).

Если $k<i<j$, то, воспользовавшись соотношением (11), получим

$$
\begin{aligned}
\alpha_{j-1} \alpha_{j-2} \cdots \alpha_{i+1} \beta_{i} \alpha_{i+1} \cdots & \\
& \alpha_{j-2} \alpha_{j-1} \alpha_{j-1} \alpha_{j-2} \cdots \alpha_{k+1} \beta_{k} \alpha_{k+1} \cdots \alpha_{j-2} \alpha_{j-1} \varepsilon_{i, k} \\
= & \varepsilon_{i, k} \alpha_{j-1} \alpha_{j-2} \cdots \alpha_{k+1} \beta_{k} \alpha_{k+1} \cdots \\
& \alpha_{j-2} \alpha_{j-1} \alpha_{j-1} \alpha_{j-2} \cdots \alpha_{i+1} \beta_{i} \alpha_{i+1} \cdots \alpha_{j-2} \alpha_{j-1} .
\end{aligned}
$$

Применив соотношения (4), сократим образуюшие $\left\{\alpha_{k}\right\}$. Пользуясь соотношениями (8), "передвинем" образующие $\left\{\alpha_{k}\right\}$ соответственно в начало и в конец соотношений и сократим их. По соотношению (11) получим равенство (19).

Если $k<j<i$, то, воспользовавшись соотношением (11), получим

$$
\begin{aligned}
\alpha_{i-1} \alpha_{i-2} \cdots \alpha_{j+1} \alpha_{j} \beta_{j} \alpha_{j} \alpha_{j+1} \cdots & \\
& \alpha_{i-2} \alpha_{i-1} \varepsilon_{k, j} \alpha_{i-1} \alpha_{i-2} \cdots \alpha_{k+1} \alpha_{k} \beta_{k} \alpha_{k} \alpha_{k+1} \cdots \alpha_{i-2} \alpha_{i-1} \\
= & \alpha_{i-1} \alpha_{i-2} \cdots \alpha_{k+1} \alpha_{k} \beta_{k} \alpha_{k} \alpha_{k+1} \cdots \\
& \alpha_{i-2} \alpha_{i-1} \varepsilon_{k, j} \alpha_{i-1} \alpha_{i-2} \cdots \alpha_{j+1} \alpha_{j} \beta_{j} \alpha_{j} \alpha_{j+1} \cdots \alpha_{i-2} \alpha_{i-1} .
\end{aligned}
$$

Сократим слева и справа образуюшие $\left\{\alpha_{k}\right\}$. Пользуясь соотношениями (8), "передвинем" образующие $\left\{\alpha_{k}\right\}$ и сократим их. По третьему равенству соотношения (8) и соотношению (11) получим соотношение (19).

Таким образом, соотношения (2), (8) выводятся из соотношений (4), $(10),(11),(12),(13)$ и могут быть исключены. Соотношение (10) является частным случаем первого равенства соотношения (11) при $j=i+1$ и тоже может быть исключено. И, наконец, в заключение доказательства исключим образующие и определяющие соотношения (11).

Равенства

$$
\sigma_{i}=\alpha_{i} \beta_{i} \quad(i=1, \ldots, n-1)
$$

показывают, каким образом автоморфизмы $\sigma_{i}$ выражаются через элементарные автоморфизмы. Равенства (20) проверяются из определений автоморфизмов. 
Tеорема 1. Группа $\mathbf{C}_{n}$ порождается автоморфизмами (3), (5) и определяющими соотношениями (4), (6), и

$$
\begin{gathered}
\alpha_{i} \sigma_{j}=\sigma_{j} \alpha_{i}, \quad|i-j|>1 \\
\sigma_{i} \alpha_{i+1} \alpha_{i}=\alpha_{i+1} \alpha_{i} \sigma_{i+1}, \quad \sigma_{i} \sigma_{i+1} \alpha_{i}=\alpha_{i+1} \sigma_{i} \sigma_{i+1} .
\end{gathered}
$$

ДокАЗАТЕЛЬСТво. По лемме 2 группа $\mathbf{C}_{n}$ порождается автоморфизмами $\left\{\alpha_{k}\right\},\left\{\beta_{k}\right\}$ и определяюшими соотношениями $(4),(12),(13)$. Добавив образуюшие (5) и определяющие соотношения (20), получим изоморффную групу. Из определений автоморфизмов $\alpha_{i}, \sigma_{i}$ следует, что равенства (6), (21) выполняются в групе $\mathbf{C}_{n}$. Следовательно, по лемме 2 они выводимы из соотношений (4), (12), (13), (20). Группа, задаваемая образуюшими $\left\{\alpha_{k}\right\},\left\{\beta_{k}\right\},\left\{\sigma_{k}\right\}$ и определяющими соотношениями (4), (6), (12), $(20),(21)$ изоморфна групше $\mathbf{C}_{n}$.

Из равенств (20) и (4) получаем

$$
\beta_{i}=\alpha_{i} \sigma_{i} \quad(i=1, \ldots, n-1) .
$$

Подставив (22) в соотношение (12), получим равенство $\alpha_{i} \sigma_{i} \alpha_{j} \sigma_{j}=$ $\alpha_{j} \sigma_{j} \alpha_{i} \sigma_{i},|i-j|>1$, вьводимое из соотношений (4), (6), (21), и, следовательно, равенство (12) можно исключить. Подставив (22) в первое, второе и третье равенства соотношений (13), получим соответственно равенства

$$
\begin{aligned}
\alpha_{i} \alpha_{j} \sigma_{j} & =\alpha_{j} \sigma_{j} \alpha_{i}, \quad|i-j|>1, \\
\alpha_{i} \alpha_{i+1} \sigma_{i+1} \alpha_{i} & =\alpha_{i+1} \alpha_{i} \sigma_{i} \alpha_{i+1} \\
\left(\alpha_{i} \alpha_{i+1} \sigma_{i+1}\right)^{2} & =\left(\alpha_{i+1} \sigma_{i+1} \alpha_{i}\right)^{2}
\end{aligned}
$$

выводимые из соотношений (4) и (21). Подставив (22) в четвертое равенство соотношений (13), получим равенство

$$
\left(\alpha_{i} \alpha_{i+1} \sigma_{i+1}\right)^{2} \alpha_{i} \sigma_{i}=\alpha_{i} \sigma_{i}\left(\alpha_{i} \alpha_{i+1} \sigma_{i+1}\right)^{2},
$$

выводимое из соотношений (21) и (6). Следовательно, соотношения (13) могут быть исключены. В заключение доказательства исключим образуюшие $\left\{\beta_{k}\right\}$ и определяюшие соотношения (20).

Лемма 3. B группе автоморфизмов $\mathbf{C}_{n}$ подгруппа $\mathfrak{M}_{n}$ пересекается с подәруппой $\mathfrak{B}_{n}$ по группе крашеных кос $\mathfrak{N}_{n}$. Образуюшие $\rho_{i, j}$ $(1 \leqslant i<j \leqslant n)$ әруппь $\mathfrak{N}_{n}$ выражсаются через образующие группы $\mathfrak{M}_{n}$ равенствами

$$
\begin{array}{r}
\rho_{i, j}=\varepsilon_{j, j-1} \varepsilon_{j, j-2} \cdots \varepsilon_{j, i+1} \varepsilon_{j, i} \varepsilon_{i, j} \varepsilon_{i+1, j} \cdots \varepsilon_{j-2, j} \varepsilon_{j-1, j} \\
(1 \leqslant i<j \leqslant n) .
\end{array}
$$


ДоКАЗАТЕЛЬСТВо. Исходя из определений (7) и пользуясь равенством

$$
\sigma_{i}=\alpha_{i} \varepsilon_{i, i+1} \quad(i=1, \ldots, n-1),
$$

выразим автоморфизмы $\rho_{i, j}$ через автоморфизмы $\left\{\varepsilon_{i, j}\right\},\left\{\alpha_{k}\right\}$. При помощи соотношений (8) получим равенства (23). Следовательно, $\mathfrak{N}_{n} \subset \mathfrak{M}_{n}$. Нити произвольной косы $C\left(\sigma_{1}, \ldots, \sigma_{n-1}\right) \in \mathfrak{B}_{n}$ реализуют подстановку $C\left(\alpha_{1}, \ldots, \alpha_{n-1}\right)$. Если $C\left(\sigma_{1}, \ldots, \sigma_{n-1}\right) \in \mathfrak{M}_{n}$, то автоморфизм $C\left(\alpha_{1}, \ldots, \alpha_{n-1}\right)$ является сопрягающим базис, и, следовательно, единичным. Таким образом, коса $C\left(\sigma_{1}, \ldots, \sigma_{n-1}\right)$ реализует единичную подстановку, т.е. является крашеной.

Теорема 2. Группа $\mathfrak{M}_{n}$ имеет тривиальный центр. Группа $\mathbf{C}_{n}$ имеет тривиальный иентр.

ДокАЗАТЕЛЬСТво. Пусть автоморфизм

$$
C=a_{i} \rightarrow X_{i}^{-1} a_{i} X_{i} \quad(i=1, \ldots, n)
$$

является элементом центра группы $\mathfrak{M}_{n}$. Докажем,что если $X-$ несократимое слово в алфавите $a_{1}, \ldots, a_{n}$ и $X^{\varepsilon_{1,2}}=X$, то слово $X$ не содержит буквы $a_{1}$. Действительно, пусть

$$
X \mp K_{1} T_{1} K_{2} T_{2} \cdots K_{s} T_{s} K_{s+1} \quad(s \geqslant 0),
$$

где $T_{1}, T_{2}, \ldots, T_{s}$ - непустые слова в алфавите $\left\{a_{3}, \ldots, a_{n}\right\}, K_{1}, K_{2}, \ldots, K_{s}$, $K_{s+1}$ - слова в алфавите $\left\{a_{1}, a_{2}\right\}$ и $K_{2}, \ldots, K_{s}$ - непустые. Легко видеть, что

$$
X^{\varepsilon_{1,2}}=K_{1}^{\varepsilon_{1,2}} T_{1} K_{2}^{\varepsilon_{1,2}} T_{2} \cdots K_{s}^{\varepsilon_{1,2}} T_{s} K_{s+1}^{\varepsilon_{1,2}}
$$

и $K_{i}^{\varepsilon_{1,2}}=a_{2}^{-1} K_{i} a_{2}$ в свободной групше. Следовательно,

$$
X^{\varepsilon_{1,2}}=a_{2}^{-1} K_{1} a_{2} T_{1} a_{2}^{-1} K_{2} a_{2} T_{2} \cdots a_{2}^{-1} K_{s} a_{2} T_{s} a_{2}^{-1} K_{s+1} a_{2},
$$

где все слова $T_{i}$ непустые, и поэтому равенство $X^{\varepsilon_{1,2}}=X$ возможно только в случае, когда $K_{i}=a_{2}^{-1} K_{i} a_{2}$, т.е., когда $K_{i}$ не содержит букв $a_{1}$. Поскольку $\varepsilon_{1,2}^{-1} C \varepsilon_{1,2}=C$, и автоморфизм $\varepsilon_{1,2}^{-1} C$ имеет вид

$$
\begin{aligned}
a_{1} & \rightarrow X_{2}^{-1} a_{2} X_{2} X_{1}^{1} a_{1} X_{1} X_{2}^{-1} a_{2}^{-1} X_{2}, \\
a_{i} & \rightarrow X_{i}^{-1} a_{i} X_{i} \quad(i=2, \ldots, n),
\end{aligned}
$$

то по доказанному подслова $X_{2}, \ldots, X_{n}$ не содержат буквы $a_{1}$. Аналогично, из равенства $\varepsilon_{2,3}^{-1} C \varepsilon_{2,3}=C$ получим, что слова $X_{1}, X_{3}, \ldots, X_{n}$ не содержат буквы $a_{2}$ и т.д. Таким образом, для любого $i=1, \ldots, n$ слово $X_{i}$ может содержать только букву $a_{i}$. Следовательно, автоморфизм $C$ тождественный. 
Пусть $C$ - элемент центра грушы $\mathbf{C}_{n}$. Пользуясь леммой 1 и соотношениями (8), представим элемент $C$ в виде $C=P\left(\ldots, \varepsilon_{i, j}, \ldots\right) Q\left(\ldots, \alpha_{i}, \ldots\right)$. Для произвольного $K\left(\ldots, \alpha_{i}, \ldots\right)$ из $\mathfrak{S}_{n}$ имеем

$$
\begin{aligned}
P\left(\ldots, \varepsilon_{i, j}, \ldots\right) Q(\ldots, & \left.\alpha_{i}, \ldots\right) K\left(\ldots, \alpha_{i}, \ldots\right) \\
& =K\left(\ldots, \alpha_{i}, \ldots\right) P\left(\ldots, \varepsilon_{i, j}, \ldots\right) Q\left(\ldots, \alpha_{i}, \ldots\right) .
\end{aligned}
$$

Пользуясь соотношениями (8), получим равенство

$$
\begin{aligned}
P\left(\ldots, \varepsilon_{i, j}, \ldots\right) Q(\ldots, & \left.\alpha_{i}, \ldots\right) K\left(\ldots, \alpha_{i}, \ldots\right) \\
& =P_{1}\left(\ldots, \varepsilon_{i, j}, \ldots\right) K\left(\ldots, \alpha_{i}, \ldots\right) Q\left(\ldots, \alpha_{i}, \ldots\right)
\end{aligned}
$$

для некоторого слова $P_{1}\left(\ldots, \varepsilon_{i, j}, \ldots\right)$. Следовательно, автоморфизм $K\left(\ldots, \alpha_{i}, \ldots\right) Q\left(\ldots, \alpha_{i}, \ldots\right) K^{-1}\left(\ldots, \alpha_{i}, \ldots\right) Q^{-1}\left(\ldots, \alpha_{i}, \ldots\right)$, сопрягающий базис, а значит тождественный, т.е. слово $Q\left(\ldots, \alpha_{i}, \ldots\right)$ коммутирует с произвольньм элементом $K\left(\ldots, \alpha_{i}, \ldots\right)$ в $\mathfrak{S}_{n}$.

Пусть $n>2$. Группа $\mathfrak{S}_{n}$ при $n>2$ имеет тривиальньй центр (см. [8, c. 68]). Следовательно, элемент центра $C$ имеет вид $P\left(\ldots, \varepsilon_{i, j}, \ldots\right)$. По первой части теоремы 5 подгруппа $\mathfrak{M}_{n}$ группы $\mathbf{C}_{n}$ имеет тривиальный центр. Следовательно, автоморфизм $C$ тождественньй. Пусть $n=2$. Группа $\mathbf{C}_{2}$ задается образуюшими $\alpha_{1}, \beta_{1}$ и определяющим соотношением $\alpha_{1}^{2}=1$, т.е. является свободным произведением двух неединичных групा и, следовательно, имеет тривиальный центр.

Будем говорить, что автоморфизм

$$
a_{i} \rightarrow X_{i}^{-1}\left(a_{1}, \ldots, a_{n}\right) a_{t_{i}} X_{i}\left(a_{1}, \ldots, a_{n}\right) \quad(i=1, \ldots, n)
$$

записан в нормальной форме, если для каждого $i=1, \ldots, n$ алгебраическая сумма показателей буквы $a_{t_{i}}$ в слове $X_{i}$ равна нулю. Легко видеть, что всякий автоморфизм может быть записан в нормальной форме. В дальнейшем, если не оговорено противное, будем считать, что автоморфизмы записьваются в нормальной форме.

Лемма 4. Элемент $P\left(\ldots, \varepsilon_{i, j}, \ldots\right)$ группь $\mathfrak{M}_{n}$ принадлежит $\mathfrak{M}_{n}^{\prime}$ тогда и только тогда, когда алгебрачческая сумма показателей по каждой образующей $\varepsilon_{i, j}$ в слове $Р$ равна нулю. Автоморфизм груп$n_{b l} \mathfrak{M}_{n}$

$$
a_{i} \rightarrow X_{i}^{-1}\left(a_{1}, \ldots, a_{n}\right) a_{i} X_{i}\left(a_{1}, \ldots, a_{n}\right) \quad(i=1, \ldots, n)
$$

принадлежит $\mathfrak{M}_{n}^{\prime}$ тогда и только тогда, когда каждое слово $X_{i}\left(a_{1}, \ldots, a_{n}\right)$ из (26) принадлежит коммутанту свободной zpynnbl $F_{n}$. 
ДокАзАтЕльство. Первое утверждение леммы легко следует из соотношений (2).

Известно, что элемент $X$ принадлежит коммутанту свободной грушшы $F_{n}$ тогда и только тогда, когда алгебраическая сумма показателей по каждой образующей $a_{i}$ равна нулю. Индукцией по длине слова $P\left(\ldots, \varepsilon_{i, j}, \ldots\right)$ легко показать, что алгебраическая сумма показателей по каждой образующей $\varepsilon_{i, j}$ в слове $P\left(\ldots, \varepsilon_{i, j}, \ldots\right)$ с автоморфизмом (26) совпадает с алгебраической суммой показателей при образующей $a_{j}$ в слове $X_{i}\left(a_{1}, \ldots, a_{n}\right)$. Следовательно, слово $P\left(\ldots, \varepsilon_{i, j}, \ldots\right)$ принадлежит $\mathfrak{M}_{n}^{\prime}$ тогда и только тогда, когда в каждом слове $X_{i}\left(a_{1}, \ldots, a_{n}\right)$ алгебраическая сумма показателей по каждой образующей $a_{j}$ при $j \neq i$ равна нулю. Поскольку автоморфизм (26) записан в нормальной форме, то алгебраическая сумма показателей по образующей $a_{i}$ тоже равна нулю.

Лемма 5. Элемент $P\left(\ldots, \alpha_{k}, \ldots, \varepsilon_{i, j}, \ldots\right)$ группы $\mathbf{C}_{n}$ принадлежит $\mathbf{C}_{n}^{\prime}$ тогда $и$ только тогда, когда слово $P$ содержит четное число букв алфавита $\left\{\alpha_{k}\right\}$ и поровну положсительных $и$ отрицательных букв алфавита $\left\{\varepsilon_{i, j}\right\}$. Автоморфизм

$$
a_{p} \rightarrow X_{p}^{-1}\left(a_{1}, \ldots, a_{n}\right) a_{i_{p}} X_{p}\left(a_{1}, \ldots, a_{n}\right) \quad(p=1, \ldots, n)
$$

принадлежит $\mathbf{C}_{n}^{\prime}$ тогда и только тогда, когда подстановка $\left(\begin{array}{c}1 \ldots n \\ i_{1} \ldots i_{n}\end{array}\right)$ четная и слово $X_{1} X_{2} \ldots X_{n}$ содержит поровну положительных $и$ отричательных букв алфавита $\left\{a_{k}\right\}$.

ДокАЗАТЕЛьСтво. Аналогично лемме 4.

ЛЕмма 6. $\mathbf{C}_{n}^{\prime} \cap \mathfrak{B}_{n}=\mathfrak{B}_{n}^{\prime}, \mathfrak{N}_{n} \cap \mathfrak{M}_{n}^{\prime}=\mathfrak{N}_{n}^{\prime}, \mathfrak{M}_{n}^{\prime} \cap \mathfrak{B}_{n}=\mathfrak{N}_{n}^{\prime}$, $\mathfrak{M}_{n}^{\prime} \cap \mathfrak{B}_{n}^{\prime}=\mathfrak{N}_{n}^{\prime}$.

ДокАЗАтЕЛьство. Легко показать, что элемент $C\left(\ldots, \sigma_{k}, \ldots\right)$ группы $\mathfrak{B}_{n}$ принадлежит $\mathfrak{B}_{n}^{\prime}$ тогда и только тогда, когда слово $C$ содержит поровну положительных и отрищательных букв алфавита $\left\{\sigma_{k}\right\}$. Следовательно, $\mathbf{C}_{n}^{\prime} \cap \mathfrak{B}_{n} \supset \mathfrak{B}_{n}^{\prime}$. Пусть $K \in \mathbf{C}_{n}^{\prime}$ и $K \in \mathfrak{B}_{n}$. Используя равенство $\sigma_{i}=\alpha_{i} \varepsilon_{i, i+1}(i=1, \ldots, n-1)$, запишем слово $K$ в алфавите $\left\{\alpha_{k}, \varepsilon_{i, j}\right\}$ и в алфавите $\left\{\sigma_{k}\right\}$. По лемме 5 слово $K$ содержит четное число букв алфавита $\left\{\alpha_{k}\right\}$ и поровну положительных и отрицательных букв алфавита $\left\{\varepsilon_{i, j}\right\}$. Пусть $K \notin \mathfrak{B}_{n}^{\prime}$. Тогда число положительных букв алфавита $\left\{\sigma_{k}\right\}$ в слове $K$ не равно числу отрицательных букв алфавита. Следовательно, число положительных букв алфавита $\left\{\varepsilon_{i, j}\right\}$ слова $K$ не равно числу отрицательных букв алфавита $\left\{\varepsilon_{i, j}\right\}$. По лемме 1 определяющие соотношения группы $\mathbf{C}_{n}$ сохраняют количество положительных и отрицательных букв алфавита $\left\{\varepsilon_{i, j}\right\}$. Следовательно, у всех слов, равных $K$, в групе $\mathbf{C}_{n}$ число положительных букв алфавита $\left\{\varepsilon_{i, j}\right\}$ не равно числу отрицательных букв алфавита. Противоречие. Следовательно, $K \in \mathfrak{B}_{n}^{\prime}$.

Группа крашеных кос $\mathfrak{N}_{n}$ порождается образуюшими $\rho_{i, j}(1 \leqslant i<j \leqslant n)$ (см. (7)). Пусть $K\left(\ldots, \rho_{i, j}, \ldots\right)$ - слово в положительных образующих 
$\left\{\rho_{i, j}\right\}$. Пусть $L\left(\ldots, \varepsilon_{i, j}, \ldots\right)$ - результат подстановки $(23)$ в слово $K(\ldots$, $\left.\rho_{i, j}, \ldots\right)$. Из формулы $(23)$ видно, что всякий элемент $\varepsilon_{q, s}$ такой, что $q<s$, слова $L\left(\ldots, \varepsilon_{i, j}, \ldots\right)$ принадлежит слову, полученному из некоторого $\rho_{t, s}$ слова $K\left(\ldots, \rho_{i, j}, \ldots\right)$ при $t \leqslant q$, и только ему. Пусть слово $L\left(\ldots, \varepsilon_{i, j}, \ldots\right)$ содержит $\left|\varepsilon_{i, j}\right|$ букв $\varepsilon_{i, j}$, и слово $K\left(\ldots, \rho_{i, j}, \ldots\right)$ содержит $\left|\rho_{i, j}\right|$ букв $\rho_{i, j}$. Тогда

$$
\left|\varepsilon_{q, s}\right|=\left|\rho_{1, s}\right|+\left|\rho_{2, s}\right|+\cdots+\left|\rho_{q, s}\right| .
$$

Из равенства (27) индукцией по первому индексу легко доказать, что

$$
\left|\rho_{1, s}\right|=\left|\varepsilon_{1, s}\right|, \quad\left|\rho_{q, s}\right|=\left|\varepsilon_{q, s}\right|-\left|\varepsilon_{q-1, s}\right| \quad(q=2, \ldots, s-1) .
$$

Из равенства (27) следует, что набор букв $\left\{\rho_{i, j}\right\}$ в слове $K\left(\ldots, \rho_{i, j}, \ldots\right)$ однозначно определяет набор букв $\left\{\varepsilon_{i, j}\right\}$ в слове $L\left(\ldots, \varepsilon_{i, j}, \ldots\right)$. Из равенств (28) следует, что набор букв $\left\{\varepsilon_{i, j}\right\}$ в слове $L\left(\ldots, \varepsilon_{i, j}, \ldots\right)$ однозначно определяет набор букв $\left\{\rho_{i, j}\right\}$ в слове $K\left(\ldots, \rho_{i, j}, \ldots\right)$. Если $K\left(\ldots, \rho_{i, j}, \ldots\right)$ в отрицательных образуюших $\left\{\rho_{i, j}\right\}$, то для него справедливо аналогичное утверждение.

Пусть элемент $P\left(\ldots, \varepsilon_{i, j}, \ldots\right)$ группы $\mathfrak{N}_{n}$ принадлежит $\mathfrak{N}_{n}^{\prime}$. Тогда он принадлежит $\mathfrak{M}_{n}^{\prime}$, поскольку $\mathfrak{N}_{n}^{\prime} \subset \mathfrak{M}_{n}^{\prime}$. Следовательно, по лемме 4 алгебраическая сумма показателей по каждой образующей $\varepsilon_{i, j}$ в слове $P\left(\ldots, \varepsilon_{i, j}, \ldots\right)$ равна нулю. Представим элемент $P\left(\ldots, \varepsilon_{i, j}, \ldots\right)$ как слово $Q\left(\ldots, \rho_{i, j}, \ldots\right)$. Предположим, что в слове $Q\left(\ldots, \rho_{i, j}, \ldots\right)$ алгебраическая сумма показателей при некоторой образующей не равна нулю. Тогда набор положительных букв $\left\{\rho_{i, j}\right\}$ в слове $Q\left(\ldots, \rho_{i, j}, \ldots\right)$ отличается от набора отрищательных букв $\left\{\rho_{i, j}\right\}$ в слове $Q\left(\ldots, \rho_{i, j}, \ldots\right)$. Пусть $P^{*}\left(\ldots, \varepsilon_{i, j}, \ldots\right)$ - результат подстановки $(23)$ в слово $Q\left(\ldots, \rho_{i, j}, \ldots\right)$. Тогда набор положительных букв $\left\{\varepsilon_{i, j}\right\}$ слова $P^{*}\left(\ldots, \varepsilon_{i, j}, \ldots\right)$ отличается от набора отрицательных букв $\left\{\varepsilon_{i, j}\right\}$ слова $P^{*}\left(\ldots, \varepsilon_{i, j}, \ldots\right)$. Так как $P^{*}\left(\ldots, \varepsilon_{i, j}, \ldots\right)=P\left(\ldots, \varepsilon_{i, j}, \ldots\right)$ в группе $\mathfrak{M}_{n}$, определяющие соотношения (2) грушы $\mathfrak{M}_{n}$ сохраняют набор $\left\{\varepsilon_{i, j}\right\}$, а вставки и сокрашения добавляют или убавляют от положительного и отришательного набора по одной букве, то набор положительных букв $\left\{\varepsilon_{i, j}\right\}$ слова $P\left(\ldots, \varepsilon_{i, j}, \ldots\right)$ отличается от набора отрицательных букв $\left\{\varepsilon_{i, j}\right\}$ слова $P\left(\ldots, \varepsilon_{i, j}, \ldots\right)$. Противоречие. Следовательно, алгебраическая сумма показателей по каждой образующей $\rho_{i, j}$ в слове $Q\left(\ldots, \rho_{i, j}, \ldots\right)$ равна нулю.

Поскольку соотношения групшы $\mathfrak{N}_{n}[6]$ сохраняют алгебраическую сумму показателей по каждой образующей, то элемент $Q\left(\ldots, \rho_{i, j}, \ldots\right)$ группы $\mathfrak{N}_{n}$ принадлежит $\mathfrak{N}_{n}^{\prime}$ тог да и только тогда, когда алгебраическая сумма показателей по каждой $\rho_{i, j}$ в слове $Q\left(\ldots, \rho_{i, j}, \ldots\right)$ равна нулю.

Таким образом, $\mathfrak{N}_{n} \cap \mathfrak{M}_{n}^{\prime}=\mathfrak{N}_{n}^{\prime}$. Отсюда и из равенства $\mathfrak{M}_{n} \cap \mathfrak{B}_{n}=\mathfrak{N}_{n}$ (см. лемму 3) следует, что $\mathfrak{N}_{n}^{\prime}=\mathfrak{M}_{n}^{\prime} \cap \mathfrak{N}_{n}=\mathfrak{M}_{n}^{\prime} \cap\left(\mathfrak{B}_{n} \cap \mathfrak{M}_{n}\right)=\mathfrak{M}_{n}^{\prime} \cap \mathfrak{B}_{n}$, т.е. $\mathfrak{M}_{n}^{\prime} \cap \mathfrak{B}_{n}=\mathfrak{N}_{n}^{\prime}$. Так как $\mathfrak{B}_{n}^{\prime} \subset \mathfrak{B}_{n}$, то $\mathfrak{M}_{n}^{\prime} \cap \mathfrak{B}_{n}^{\prime} \subset \mathfrak{N}_{n}^{\prime}$. Поскольку $\mathfrak{N}_{n} \subset \mathfrak{M}_{n}$ и $\mathfrak{N}_{n} \subset \mathfrak{B}_{n}$, то $\mathfrak{N}_{n}^{\prime} \subset \mathfrak{M}_{n}^{\prime}$ и $\mathfrak{N}_{n}^{\prime} \subset \mathfrak{B}_{n}^{\prime}$. Следовательно, $\mathfrak{M}_{n}^{\prime} \cap \mathfrak{B}_{n}^{\prime} \supset \mathfrak{N}_{n}^{\prime}$ и $\mathfrak{M}_{n}^{\prime} \cap \mathfrak{B}_{n}^{\prime}=\mathfrak{N}_{n}^{\prime}$. 
ЛЕмма 7. Пусть $A\left(\ldots, \varepsilon_{i, j}, \ldots\right)$ является крашеной косой группь $\mathfrak{N}_{n}$. Пусть коса $B\left(\ldots, \varepsilon_{i, j}, \ldots\right)$ получена из косы $A\left(\ldots, \varepsilon_{i, j}, \ldots\right)$ выдергиванием $p$-й нити $(p=1, \ldots, n)$. Пусть слово $C\left(\ldots, \varepsilon_{i, j}, \ldots\right)$ получено из слова $A\left(\ldots, \varepsilon_{i, j}, \ldots\right)$ вычеркиванием всех букв $\varepsilon_{p, \alpha}, \varepsilon_{\alpha, p}$ $(\alpha=1, \ldots, n)$ и уменьшением на единичу всех индексов, больших $p, \quad$ во всех образуюших $\varepsilon_{i, j}$. Тогда $C\left(\ldots, \varepsilon_{i, j}, \ldots\right)$ - коса $u$ $B\left(\ldots, \varepsilon_{i, j}, \ldots\right)=C\left(\ldots, \varepsilon_{i, j}, \ldots\right)$ в zpynne $\mathfrak{N}_{n-1}$.

ДоКАЗАТЕЛЬСТВО. Пусть $\omega_{p}(p=1, \ldots, n)$ - гомоморфизм групшы $\mathfrak{N}_{n}$ в группу $\mathfrak{N}_{n-1}$, состоящий в выдергивании $p$-й нити косы. Из определения гомоморфизмов $\omega_{p}$ и формул (7) легко получить формулы действия гомоморфизмов $\omega_{p}$ на образуюших $\left\{\rho_{i, j}\right\}$ группы $\mathfrak{N}_{n}$ :

$$
\begin{aligned}
& \omega_{p}\left(\rho_{k, l}\right)=\rho_{k-1, l-1}, \quad 1 \leqslant p<k, \\
& \omega_{p}\left(\rho_{k, l}\right)=\rho_{k, l-1}, \quad k \leqslant p<l \quad(k<l-1) \text {, } \\
& \omega_{p}\left(\rho_{k, l}\right)=1, \quad k=p=l-1 \vee p=l \text {, } \\
& \omega_{p}\left(\rho_{k, l}\right)=\rho_{k, l}, \quad l<p \leqslant n .
\end{aligned}
$$

Пусть $\Psi_{p}(p=1, \ldots, n)$ - отображение грушшы $\mathfrak{M}_{n}$ в группу $\mathfrak{M}_{n-1}$, состоящее в вычеркивании всех букв $\varepsilon_{p, \alpha}, \varepsilon_{\alpha, p}(\alpha=1, \ldots, n)$ и уменьшением на единицу всех индексов, больших $p$, во всех образующих $\varepsilon_{i, j}$. Если $K\left(\ldots, \varepsilon_{i, j}, \ldots\right)$ переводится в $L\left(\ldots, \varepsilon_{i, j}, \ldots\right)$ одним элементарным преобразованием группы $\mathfrak{M}_{n}($ см. $(2))$, то $\Psi_{p} K\left(\ldots, \varepsilon_{i, j}, \ldots\right)=\Psi_{p} L\left(\ldots, \varepsilon_{i, j}, \ldots\right)$ в группе $\mathfrak{M}_{n}$. Следовательно, если $K\left(\ldots, \varepsilon_{i, j}, \ldots\right)=Q\left(\ldots, \varepsilon_{i, j}, \ldots\right)$ в группе $\mathfrak{M}_{n}$, то

$$
\Psi_{p} K\left(\ldots, \varepsilon_{i, j}, \ldots\right)=\Psi_{p} Q\left(\ldots, \varepsilon_{i, j}, \ldots\right)
$$

в групше $\mathfrak{M}_{n}$. Отображение $\Psi_{p}$, очевидно, сохраняет операцию. Следовательно, отображение $\Psi_{p}(p=1, \ldots, n)$ является гомоморфизмом.

Образующие $\rho_{k, l}(1 \leqslant k<l \leqslant n)$ группы $\mathfrak{N}_{n}$ выражаются через образуюшие группы $\mathfrak{M}_{n}$ равенствами (см. (23))

$$
\rho_{k, l}=\varepsilon_{l, l-1} \varepsilon_{l, l-2} \cdots \varepsilon_{l, k+1} \varepsilon_{l, k} \varepsilon_{k, l} \varepsilon_{k+1, l} \cdots \varepsilon_{l-2, l} \varepsilon_{l-1, l} .
$$

Из формул (29) и (30) следует, что

$$
\omega_{p}\left(\rho_{k, l}\right)=\Psi_{p}\left(\rho_{k, l}\right) \quad(p=1, \ldots, n, \quad 1 \leqslant k<l \leqslant n) .
$$

Пусть $A^{\prime}\left(\ldots, \varepsilon_{i, j}, \ldots\right)$ - представление косы $A\left(\ldots, \varepsilon_{i, j}, \ldots\right)$ в образующих (23) (см. лемму 3 ). Поскольку отображения $\omega_{p}$ и $\Psi_{p}$ суть гомоморфизмы, то из формул (31) следует, что

$$
\omega_{p} A^{\prime}\left(\ldots, \varepsilon_{i, j}, \ldots\right)=\Psi_{p} A^{\prime}\left(\ldots, \varepsilon_{i, j}, \ldots\right) .
$$

Следовательно,

$$
\begin{aligned}
B\left(\ldots, \varepsilon_{i, j}, \ldots\right) & =\omega_{p} A\left(\ldots, \varepsilon_{i, j}, \ldots\right)=\omega_{p} A^{\prime}\left(\ldots, \varepsilon_{i, j}, \ldots\right), \\
C\left(\ldots, \varepsilon_{i, j}, \ldots\right) & =\Psi_{p} A\left(\ldots, \varepsilon_{i, j}, \ldots\right)=\Psi_{p} A^{\prime}\left(\ldots, \varepsilon_{i, j}, \ldots\right) .
\end{aligned}
$$

Таким образом, $C\left(\ldots, \varepsilon_{i, j}, \ldots\right)=B\left(\ldots, \varepsilon_{i, j}, \ldots\right)$ в групше $\mathfrak{N}_{n-1}$. 
ЛЕмма 8. Пусть автоморфизм $B\left(\ldots, \varepsilon_{i, j}, \ldots\right)$ әруппы $\mathfrak{M}_{n}$ имеет вид

$$
a_{k} \rightarrow Y_{k}^{-1} a_{k} Y_{k} \quad(k=1, \ldots, n),
$$

$u$ пусть $p$ - фиксированное число $(p=1, \ldots, n)$. Пусть слово $C\left(\ldots, \varepsilon_{i, j}, \ldots\right)$ получено из слова $B\left(\ldots, \varepsilon_{i, j}, \ldots\right)$ вычеркиванием всех букв $\varepsilon_{p, \alpha}, \varepsilon_{\alpha, p}(\alpha=1, \ldots, n)$, и пусть автоморфизм $C\left(\ldots, \varepsilon_{i, j}, \ldots\right)$ имеет вид

$$
a_{k} \rightarrow Z_{k}^{-1} a_{k} Z_{k} \quad(k=1, \ldots, n) .
$$

Тогда слово $Z_{p}$ пусто, а всякое слово $Z_{k}(k \neq p)$ получено из слова $Y_{k}$ вычеркиванием всех вхождений буквы $a_{p}^{ \pm 1}$.

ДокАЗАТЕЛЬСТво. Индукция по длине слова $B\left(\ldots, \varepsilon_{i, j}, \ldots\right)$. Если длина слова $B\left(\ldots, \varepsilon_{i, j}, \ldots\right)$ равна нулю, то длина всякого слова $Y_{i}$ равна нулю, и лемма очевидна. Пусть $B \mp B_{1} \varepsilon_{s, t}^{ \pm 1}$, автоморфизм $B$ имеет вид (32), а автоморфизм $B_{1}$ имеет вид

$$
a_{k} \rightarrow W_{k}^{-1} a_{k} W_{k} \quad(k=1, \ldots, n) .
$$

По определению $\varepsilon_{s, t}^{ \pm 1}$ всякое $Y_{k}$ из (32) при $k \neq s$ получается из $W_{k}$ и (34) заменой $a_{s} \rightarrow\left(a_{t}\right)^{\mp 1} a_{s} a_{t}^{ \pm 1}$, а при $k=s$ слово $Y_{k}$ имеет вид $a_{t}^{ \pm 1} Y_{k}^{\prime}$, где $Y_{k}^{\prime}$ получается из $W_{k}$ заменой $a_{s} \rightarrow\left(a_{t}\right)^{\mp 1} a_{s} a_{t}^{ \pm 1}$.

Если $s \neq p, t \neq p$, то $C=C_{1} \varepsilon_{s, t}^{ \pm 1}$, где слово $C_{1}$ получено из $B_{1}$ вычеркиванием всех $\varepsilon_{p, \alpha}, \varepsilon_{\alpha, p}(\alpha=1, \ldots, n)$. По индуктивному предположению автоморфизм имеет вид

$$
a_{k} \rightarrow V_{k}^{-1} a_{k} V_{k} \quad(k=1, \ldots, n),
$$

где слово $V_{p}$ пусто, а всякое слово $V_{k}(k \neq p)$ получено из слова $W_{k}$ вычеркиванием всех вхождений буквы $a_{p}^{ \pm 1}$. В свободной груше $F_{n}$ слово, в котором сначала вычеркивается буква $a_{p}^{ \pm 1}$, а затем производится замена $a_{s} \rightarrow\left(a_{t}\right)^{\mp 1} a_{s} a_{t}^{ \pm 1}(s \neq p, t \neq p)$ равно слову, в котором сначала производится указанная замена, а затем вычеркивается буква $a_{p}^{ \pm 1}$. Таким образом, в рассматриваемом случае лемма верна.

Пусть $s=p$, т.е. $B \mp B_{1} \varepsilon_{p, t}^{ \pm 1}$. По индуктивному предположению автоморфизм слова $C_{1}$, полученного из слова $B_{1}$ вычеркиванием всех $\varepsilon_{p, \alpha}, \varepsilon_{\alpha, p}(\alpha=1, \ldots, n)$ имеет вид $(35)$, где слово $V_{p}$ пусто, а всякое слово $V_{k}(k \neq p)$ получено из слова $W_{k}$ вычеркиванием всех вхождений буквы $a_{p}^{ \pm 1}$. По условию в данном случае $C=C_{1}$. Следовательно, $Z_{p}$ пусто, а $Z_{k}(k \neq p)$ получено из слова $W_{k}$ вычеркиванием всех букв $a_{p}^{ \pm 1}$. Слово $Y_{k}$ получается из слова $W_{k}$ заменой $a_{p} \rightarrow\left(a_{t}\right)^{\mp 1} a_{p} a_{t}^{ \pm 1}$. Следовательно, результат вьчеркивания всех букв $a_{p}^{ \pm 1}$ в словах $W_{k}$ и $Y_{k}(k \neq p)$ совпадают. Таким образом, слово $Z_{k}(k \neq p)$ получено из слова $Y_{k}$ вычеркиванием всех вхождений буквы $a_{p}^{ \pm 1}$. 
Пусть $t=p$, т.е. $B \mp B_{1} \varepsilon_{s, p}^{ \pm 1}$. По индуктивному предположению автоморфизм слова $C_{1}$, полученного из слова $B_{1}$ вычеркиванием всех $\varepsilon_{p, \alpha}, \varepsilon_{\alpha, p}$ $(\alpha=1, \ldots, n)$, имеет вид $(35)$, где $V_{p}$ пусто, а всякое $V_{k}(k \neq p)$ получено из слова $W_{k}$ вычеркиванием всех вхождений буквы $a_{p}^{ \pm 1}$. В данном случае $C=C_{1}$. Следовательно, $Z_{p}$ пусто, а $Z_{k}(k \neq p)$ получено из слова $W_{k}$ вычеркиванием всех вхождений буквы $a_{p}^{ \pm 1}$. Слово $Y_{k}(k \neq p)$ получается из слова $W_{k}$ заменой $a_{s} \rightarrow\left(a_{p}\right)^{\mp 1} a_{s} a_{p}^{ \pm 1}$. Следовательно, результаты вычеркивания буквы $a_{p}^{ \pm 1}$ в словах $W_{k}$ и $Y_{k}(k \neq p)$ совпадают. Таким образом, $Z_{k}(k \neq p)$ получается из слова $Y_{k}$ вычеркиванием всех вхождений букв $a_{p}^{ \pm 1}$.

Коса $A$ групшы кос $\mathfrak{B}_{n}$ назьвается гладкой, если выдергивание всякой нити из $A$ переводит еев косу, равную единище в $\mathfrak{B}_{n-1}$ (см. [9, 6.23]). Легко видеть, что при $n>2$ гладкая коса принадлежит $\mathfrak{N}_{n}$.

Пусть $Z_{n}=\left\langle t_{1}, \ldots, t_{n}\right\rangle$ - свободная абелева групша ранга $n$ со свободным базисом $t_{1}, \ldots, t_{n}, J Z_{n}$ - групповое кольцо групшы $Z_{n}$ и кольца целых чисел $J$. Представление Гасснер [10] группы крашеных кос $\mathfrak{N}_{n}$ в мультипликативную группу $n \times n$ матрищ с элементами из $J Z_{n}$ - это гомоморфизм группы $\mathfrak{N}_{n}$, определяемьй отображением образуюших группы $\mathfrak{B}_{n}$ (см. (7))

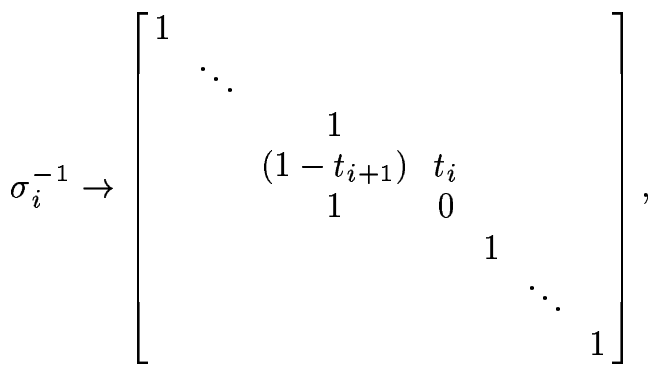

где выделены $i$-я строка и $(i+1)$-я строка.

Магнус и Пелузо [11] доказали, что представление Гасснер для $\mathfrak{N}_{3}$ точное. Бирман (см. [7, теорема 3.14]) показала, что ядро представления Гасснер группы $\mathfrak{N}_{n}$ принадлежит $\mathfrak{N}_{n}^{\prime}$.

ТЕОРема 3. Всякий әлемент из ядра представления Гасснер группы $\mathfrak{N}_{n}$ при выдергивании любой нити переходит в некоторый әлемент ядра представления Гасснер группы $\mathfrak{N}_{n-1}$. В частности, если представление Гасснер группы $\mathfrak{N}_{n-1}$ точное, то ядро представления Гасснер группы $\mathfrak{N}_{n}$ содержится в группе гладких кос с $n$ нитями $\Gamma_{n}$.

ДоКАЗАТЕЛЬСТво. Легко видеть, что если $K_{1}\left(a_{1}, \ldots, a_{n}\right)=K_{2}\left(a_{1}\right.$, $\left.\ldots, a_{n}\right)$ в свободной группе, то слова $L_{1}\left(a_{1}, \ldots, a_{n-1}\right)$ и $L_{2}\left(a_{1}, \ldots, a_{n-1}\right)$, полученные соответственно из слов $K_{1}$ и $K_{2}$ вычеркиванием всех вхождений буквы $a_{p}^{ \pm 1}$ и уменьшением на единицу всех индексов в буквах $a_{p+1}, \ldots, a_{n}$ равны в группе. Таким образом, при этом отображении каждьй коммутатор групшы $F_{n}$ переходит в некоторьй коммутатор 
группы $F_{n-1}$, а всякий элемент коммутанта групшы $F_{n}$ переходит в некоторый элемент коммутанта групшы $F_{n-1}$. В свою очередь, всякий элемент $F_{n}^{\prime \prime}$ переходит в некоторьй элемент $F_{n-1}^{\prime \prime}$.

Таким образом, если $X\left(a_{1}, \ldots, a_{n}\right)$ - элемент $F_{n}^{\prime \prime}$ и $Y\left(a_{1}, \ldots, a_{n-1}\right)$ слово, полученное из слова $X\left(a_{1}, \ldots, a_{n}\right)$ вычеркиванием всех вхождений буквы $a_{p}^{ \pm 1}$ и уменьшением на единицу всех индексов в буквах $a_{p+1}, \ldots, a_{n}$, то $Y\left(a_{1}, \ldots, a_{n-1}\right)$ принадлежит $F_{n-1}^{\prime \prime}$.

Пусть коса $A$ - элемент ядра представления Гасснер группы $\mathfrak{N}_{n}$. Бирман (см. [7, теорема 3.16]) показала, что автоморфизм $A=a_{k} \rightarrow X_{k}^{-1} a_{k} X_{k}$ $(k=1, \ldots, n)$ группы крашеных кос $\mathfrak{N}_{n}$ (записанньй в нормальной форме) принадлежит ядру представления Гасснер группы $\mathfrak{N}_{n}$ тогда и только тогда, когда каждое слово $X_{i}$ принадлежит $F_{n}^{\prime \prime}$. Выдернем в косе $A p$-ю нить $(p=1, \ldots, n)$. Получим косу $B$ с автоморфизмом $a_{k} \rightarrow Y_{k}^{-1} a_{k} Y_{k}$ $(k=1, \ldots, n)$. По леммам 7,8 слово $Y_{p}$ пусто, а каждое слово $Y_{k}(k \neq p)$ получено из слова $X_{k}$ вычеркиванием всех вхождений буквы $a_{p}^{ \pm 1}$ и уменьшением на единицу всех индексов в буквах $a_{p+1}, \ldots, a_{n}$. По доказанному $Y_{k}\left(a_{1}, \ldots, a_{n-1}\right) \in F_{n}^{\prime \prime}$. По теореме Бирман коса $B$ принадлежит ядру представления Гасснер группы $\mathfrak{N}_{n-1}$, т.е. по условию теоремы $B=1$ в группе $\mathfrak{N}_{n-1}$. Таким образом, выдергивание $p$-й нити $(p=1, \ldots, n)$ переводит косу $A$ в единичную, т.е. $A \in \Gamma_{n}$.

Пусть $Z=\langle t\rangle-$ бесконечная циклическая группа и $J Z-$ групповое кольцо групшы $Z$ и кольца целых чисел $J$. Представление Бурау (см., например, $\left[7\right.$, c. 118]) группы кос $\mathfrak{B}_{n}$ в мультипликативную группу $n \times n$ матриц с элементами из $J Z$ - это гомоморфизм, определяемьй отображением

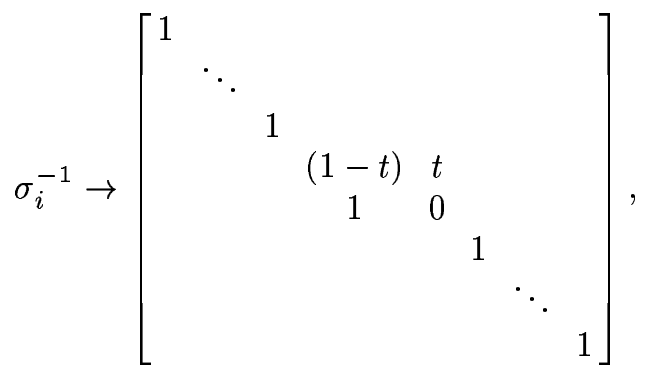

где выделены $i$-я строка и $(i+1)$-я строка.

Магнус и Пелузо [11] доказали, что представление Бурау для $\mathfrak{B}_{3}$ точное. Бирман (см. [7, теорема 3.14]) показала, что ядро представления Бурау группы кос $\mathfrak{B}_{n}$ принадлежит $\mathfrak{B}_{n}^{\prime} \cap \mathfrak{N}_{n}$.

ТЕОРемА 4. Ядро представления Бурау группы кос $\mathfrak{B}_{n}$ принадлежum $\mathfrak{N}_{n}^{\prime}$.

ДоКАЗАТЕЛЬСТВо. Пусть $H_{n}$ - подгруппа свободной групшы $F_{n}$, состоящая из тех слов в алфавите $a_{1}^{ \pm 1}, \ldots, a_{n}^{ \pm 1}$, которые содержат поровну положительных и отрищательных букв. Поскольку $H_{n}$ - подгруппа груп- 
пы $F_{n}$, то $H_{n}^{\prime}$ - подгруппа группы $F_{n}^{\prime}$. Бирман (см. [7, теорема 3.16$\left.]\right)$ показала, что автоморфизм

$$
A=\left\{\begin{array}{l}
a_{1} \rightarrow X_{1}^{-1} a_{1} X_{1} \\
\ldots \ldots \ldots \cdots \\
a_{n} \rightarrow X_{n}^{-1} a_{n} X_{n}
\end{array}\right.
$$

группы крашеных кос $\mathfrak{N}_{n}$ (записанньй в нормальной форме) принадлежит ядру представления Бурау группы $\mathfrak{B}_{n}$ тогда и только тогда, ког да каждое слово $X_{i}$ автоморфизма $A\left(\ldots, \varepsilon_{i, j}, \ldots\right)$ принадлежит $H_{n}^{\prime}$. Следовательно, если $A\left(\ldots, \varepsilon_{i, j}, \ldots\right)$ принадлежит ядру представления Бурау групшы $\mathfrak{B}_{n}$, то каждое слово $X_{i}$ автоморфизма $A\left(\ldots, \varepsilon_{i, j}, \ldots\right)$ принадлежит $F_{n}^{\prime}$, откуда по лемме 4 получаем, что $A\left(\ldots, \varepsilon_{i, j}, \ldots\right)$ принадлежит $\mathfrak{M}_{n}^{\prime}$. По теореме 3.14 работы [7] автоморфизм $A\left(\ldots, \varepsilon_{i, j}, \ldots\right)$ принадлежит $\mathfrak{B}_{n}^{\prime}$. По лемме 6 групша $\mathfrak{M}_{n}^{\prime}$ пересекается с группой $\mathfrak{B}_{n}^{\prime}$ по подгрупе $\mathfrak{N}_{n}^{\prime}$. Таким образом, ядро представления Бурау группы кос $\mathfrak{B}_{n}$ принадлежит $\mathfrak{N}_{n}^{\prime}$.

\section{СПИСОК ЦИТИРОВАННОЙ ЛИТЕРАТУРЫ}

[1] McCool J. On basis-conjugating automorphisms of free groups // Canad. J. Math. 1986. V. 38. №6. P. 1525-1529.

[2] Humphries S.P. On weakly distinguished bases and free generating sets of free group // Quart. J. Math. 1985. V. 2. № 36. P. 215-219.

[3] Coxeter H.S., Moser W. O. Generators and relations for discrete groups. New York: Springer Verlag, 1972.

[4] Магнус В., Каррас А., Солитер Д. Комбинаторная теория групп. М.: Наука, 1974.

[5] Artin E. Theorie der Zopfe. Sem. Univ. Hamburg // Abh. Math. 1926. № 4. P. 47-72.

[6] Марков А.А. Основы алгебраической теории кос // Тр. МИАН. 1945. № 16 . C. $1-53$.

[7] Birman J. Braids, Links, Mapping Class Groups // Ann. Math. Studies. V. 82. Princeton: Princeton Univ. Press, 1975.

[8] Курош А. Г. Теория групп. М.: Наука, 1967.

[9] Коуровская тетрадь. (Нерешенные вопросы теории групп). Новосибирск: Наука, 1984. 9-е изд.

[10] Gassner B. J. On braid group. Sem. Univ. Hamburg // Abh. Math. 1961. V. 25. P. $10-22$.

[11] Magnus W., Peluso A. On knot groups // Comm. Pure Appl. Math. 1967. V. 20. P. $749-770$. 